\title{
The Science and Technology of Manuscript Writing-aid and Folk Paintings in Medieval Assam^
}

\author{
Robin Kumar Dutta ${ }^{\mathrm{a}, *}$, Barsha R. Goswami ${ }^{\mathrm{b}}$, Niranjan Ligira ${ }^{\mathrm{c}}$ \\ ${ }^{a}$ Professor, ${ }^{b}$ Research Scholar, ${ }^{c}$ Post Graduate Student \\ Department of Chemical Sciences, Tezpur University, Assam.
}

\section{Introduction}

The project aimed to study the previously unexplored unique indigenous technologies of medieval Assam, namely, preparation of the traditional writing-aids of Sancipat, the writing base; and scaling up of Mahī, the herbal ink. It also focused on the methods of painting of manuscripts, artifacts and handicrafts to understand the traditional scientific and technological aspects involved in them and scale-up the technologies for possible tourism marketing. The study involved three components, (i) to study surface-active property, method of preparation and improvement in the properties of Mahi, (ii) understanding the traditional method of preparation and preservation of Sancipat and improvement in physical and chemical behavior of Sancipat (Agaru-bark) at various stages of preparation, physicochemical and biochemical properties such as fluorescence, antifungal etc. (iii) to study the methods of preparation and application of the herbal dyes and mineral pigments for illustration of Sancipat manuscript and wooden statues in Sattras.

\section{Tradition of manuscript writing in}

\section{Assam}

There was a unique and splendid tradition of manuscript writing along with manuscript illustration in Assam on Sancipat, a writing base made of tree bark called Sanci (Agaru, Aquilaria agalocha) with Mahī, an herbal ink

DOI: $10.16943 / \mathrm{ijhs} / 2020 / v 55 i 4 / 158292$

*Email: robind@tezu.ernet.in.

${ }^{\wedge}$ The project was accomplished under the sponsorship of Indian National Commission for History of Science between the period July 2017 and June 2019. which started by the 7th century and continued till the early 20th century (Figure 1) (Gait 1906, Kalita, 1990). The tradition was taken to its peak by the Vaishnavite movement in Assam lead by Shrimanta Sankardeva in the 16th century BCE. Tens of thousands of such centuries old manuscripts are still available in Assam. In addition to those in Assam, hundreds of such manuscripts were also found in other adjoining areas like North Bengal and northern Bangladesh. The Sancipat manuscripts have tremendous literary values in addition to religious, cultural and historical importance. While paper made of cotton (tulapat), cloth, clay, metals like copper, stone, brick, wooden board, bamboo sheet, palm leaves, animal skins, etc., were used as writing base in other parts of the world, Sancipat was popular in Assam. The making of Sancipat from the bark of Sanci (Agaru, Aquilaria agallocha) tree involved an arduous process involving curing, seasoning and polishing raw slices of bark before it could be made to retain the ink.

The Assamese traditional ink, Mahī, which shows no sign of corrosion was prepared from mainly fruit pulp of Hilikha (Terminalia chebula) and some ingredients of herbal and animal origin with cow-urine as an extractant to facilitate the extraction of the dyes from the plant sources. The indigenous ink, Mahī, used for writing also was prepared from locally available herbal ingredients through a unique method not known in any other part of the world. Some other herbal and mineral paints as vermilion (hengul in Assamese, hingula in Sanskrit), Yellow ochre (hāitāl in Assamese, harîtāla in Sanskrit) together with herbal Indigo (nīla) for blue, kharimātī or dhālmātī for white, Carbon black (kājala), Calcium carbonate or sulphate (Chalk) etc which stands out both for the brightness of their hues were also used for painting the manuscripts. The survival of the Sancipat manuscripts 


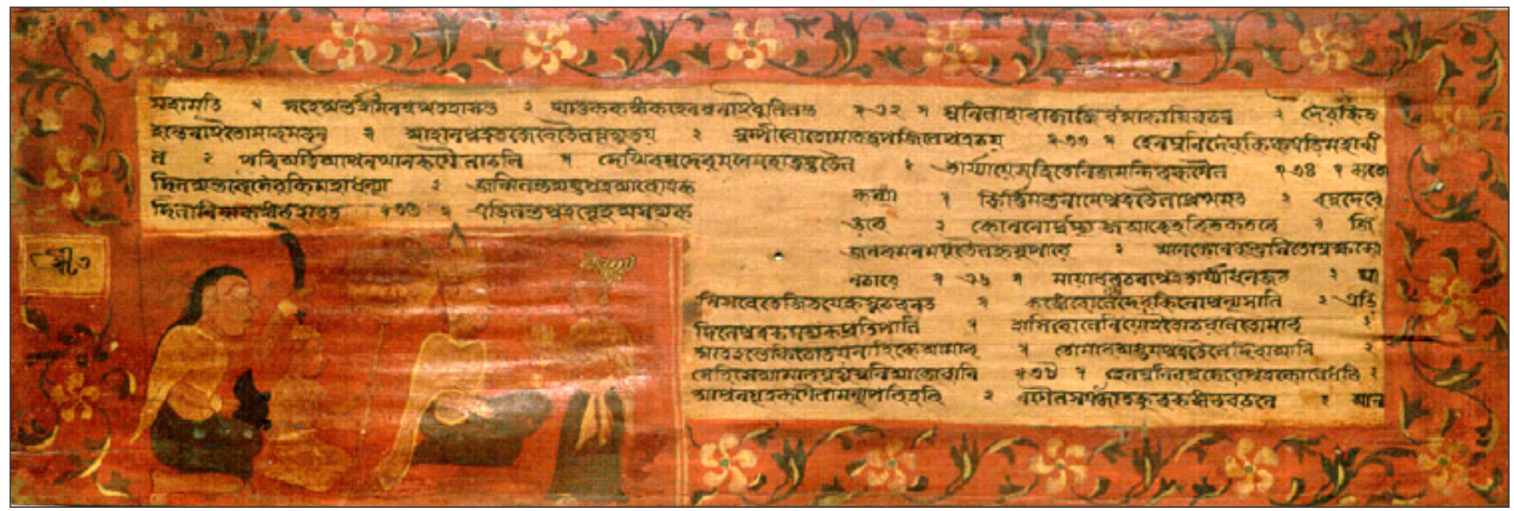

Figure 1 A page from Adi Dasama, an illustrated manuscript of Sancipat copied in 1799 CE and preserved in Bengenaati Satra, Majuli

for centuries in the hot and humid climate of Assam may also points to possible contributions from hengul and $h \bar{a} i t a \bar{l} l$ used in the manuscripts. Thus, it is interesting to note the survival of Sancipat manuscripts with its allure finish for centuries despite an unfavourable hot and humid climate that incubates fungus and insect which feed on cellulosic materials like Sancipat.

Though there are some minor variations in the exact procedures of preparation of Sancipat, the principle is essentially the same and involves the major steps, such as acquiring, cleaning, cutting into required size, partial degumming, treatment for paste-resistance, smoothening and finally coating with hāitāl and/or hengul. The coat of hâitāl and hengul (cinnabar) may also protect the Sancipat from paste and fungus. Two research articles on these has drawn attention of people from different walks of life (Sah 2006, Nath 2015, pp. 207-238, Dutta 2015, 239-261).

Through this project we looked for the possible factors that have helped the Sancipat manuscripts to survive over centuries in the harsh climate. For that, we looked at the chemistry of the method of preparation of Sancipat and Mahī; and the pigments used in preparation of Sancipat and in the illustrations in the manuscripts. Some herbal and mineral paints were also used for painting manuscripts, masks used in drama and other traditional artifacts like statues. A plan to study the chemistry of these paints and their application was organized along with the possible health effects of these indigenous techniques with regard to toxicity. These may lead to more interesting findings about the traditional manuscript writing technology, indigenous preservation methods of early Assam. It may help promote commercialisation of handicrafts production for tourism marketing as it will throw light on dying Sancipat making process which once bloomed to full extent as mentioned in Assamese literature. It is worth mentioning that the study of manuscripts or research activities related to the traditional manuscripts are as low as negligible. A systematic study will help us in knowing the traditions which have historical, literary and cultural importance. The proposed work has interdisciplinary relevance involving chemistry, biochemistry, physics, indigenous technology, history, literature and traditional culture and heritage. It would also create public awareness. The work was carried out under the following chapters:

1. Methodologies used for the study

2. Findings and Discussion (i) Scientific study of Mahī (ii) Scientific study of Sancipat (iii) Reviving the dyeing traditions of Sancipat and hengul-hāitāl

\section{Methodologies used for the study}

The methodology involved collection of information available in the literature on preparation and application methods. The information from the few people who are still practicing these methods was collected through field visit and had a hands-on training on the traditional method of manuscript preparation by organizing a small workshop. The detailed methodologies followed for individual objectives are described below: 


\subsection{Study of surface active property of Mahī}

The durability of Mahi has been considered to be its major attributes that attracts the attention of many historians, curators and traditional practitioners. Surface active property of Mahi and the role of biosurfactants as green stabilizer on the chromogenic components of Mahi have been studied. The color intensification has been studied through interactions of two representative polyphenols present in $M a h \bar{l}$, viz., gallic acid and quercetin with iron forming iron-polyphenol complexes, and that of the complexes with surfactants of various charge types (Figure 2).

\subsection{Scaling up of preparation of Mahī}

An attempt has been made to improve the traditional procedure at artificial setting under refrigerator instead of natural setting in cold condition. Here, we have successfully optimized the cold extraction of Mahī. As regards, the choice of using analytical grade $\mathrm{FeSO}_{4}$ (II) as mordanting agent in place of rusted iron nails, to standardize the amount of $\mathrm{Fe}$ (II) to get intense black coloured ink, the concentration of $\mathrm{Fe}$ (II) was increased ranging from 0 $300 \mathrm{ppm}$ and observed that $300 \mathrm{ppm}$ of $\mathrm{Fe}$ (II) is optimum concentration for getting instant black color in Mahī. Different analytical and spectrophotometric techniques viz.; UV-Vis, FT-IR, Raman, XRD were used to analyze the quality of the ink.

\subsection{Study of Sancipat, the writing base}

The writing base Sancipat was prepared by following the traditional method (Figure 3). Variation of ingredients was done to see the effects and contributions of individual ingredients. The physical and chemical behavior of Agaru-bark at various stages of preparation of Sancipat was studied by chemical and physical analysis and comparison between a bark and a manuscript paper with respect to various physicochemical parameters including tensile strength (by Universal tensile machine), glossiness (by Glossmeter), etc. The biochemical properties of Sancipat was studied by antifungal assay. Another work on the scaling up of preparation of Sancipat was done by studying the treatments of Sancipat with different herbal and mineral and manipulating the operational parameters like pressing, heating, etc. Value addition to the product was done by optimizing the quality-determining pa- rameters.

\subsection{Study of application of herbal and mineral paints and pigments}

The methods of preparation and application of the herbal dyes and mineral pigments along with various natural gums and other additives for painting artifacts and handicrafts were studied. One work on restoration of old wooden sculptures was carried out in the form of workshop. The purpose of these restoration workshops was to carry out practical study and train people in the tradition on along with the restoration of the wearing out centuriesold wooden artefacts.

\section{Findings and Discussion}

This project work focused on different areas like scientific study of Mahī, Sancipat and reviving the dyeing traditions of Sancipat and hengul-hāitāl.

\subsection{Scientific Study of Mahī}

This detailed study on, Mahī, presents some interesting physicochemical behavior vis-à-vis its possible phytochemical constituents. We have identified some polyphenolic chemicals responsible for the color of Mahī. From our recent study, the presence of phenolic acids (Gallic acid and Ellagic acid), four flavonoids (Epigallocatechin gallate, Quercetin, Kaempferol and Epicatechin) and one tannin (Tannic acid) in Mahi samples have been confirmed by HPLC analysis. Another observation was that Raman analysis of Mahi failed at $514 \mathrm{~nm}$ excitation, indicative of increased production of fluorescence of the Mahi samples around this region that obscure the Raman signals. The observed fluorescence of Mahi has been attributed to the phenolic acids, flavonoids and chlorophyll pigments. UV fluorescence images along with images taken by fluorescence microscope under RFP filter conforms auto-fluorescence of Mahì.

In the present study, we have analyzed some physicochemical and biochemical properties of freshly prepared Mahi to know the reasons for the glaze and stability of the ink particles for centuries without adding some binder. First, we established a comparision of content determination of total saponins among the mostly used herbal ingredients. After that, we studied the effect of complexation 

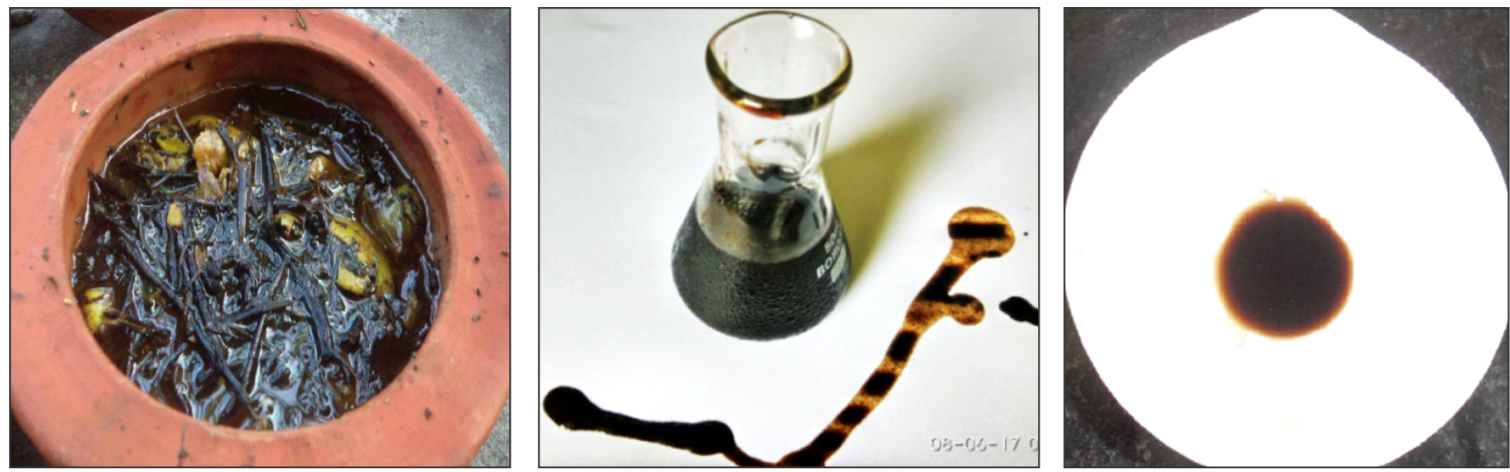

Figure 2 Preparation of $M a h \bar{\imath}$ in earthen pot by traditional method and samples in glass and porcelain containers.
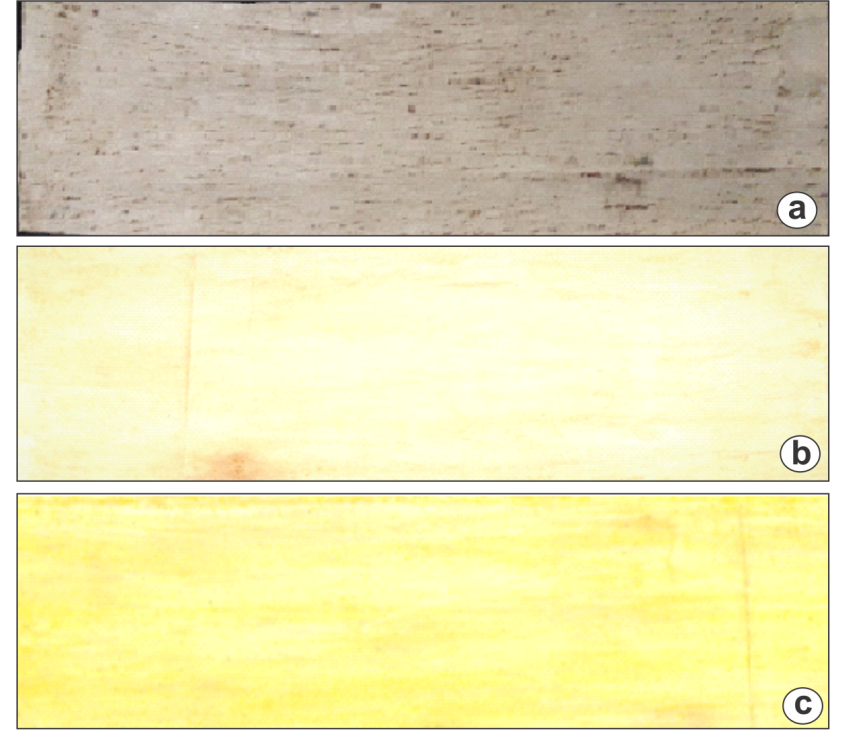

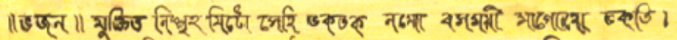

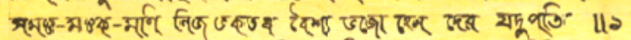

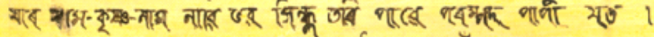

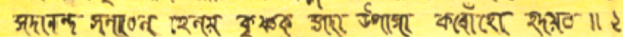

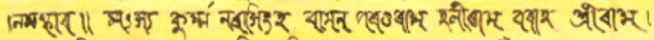

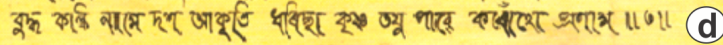

Figure 3 A strip of Sanci bark at various stages of traditional method of preparation: (a) after degumming, (b) Manuscript after fatty pulse treatment (c) Haritāla applied on Sancipāt manuscript and (d) Sancipāt manuscript decorated with thin coat of hengul on the outer border and inscribed with herbal ink, Māhi under supervision of a traditional practitioner.

of both Iron (II)-Gallic acid and Iron (II)-Quercetin complexes in aqueous solution and in micellar solutions of an- ionic surfactant with emphasis on the UV-Visible spectra and stoichiometry by Job's method of both the complexes. From the study, we observed the effects of anionic surfactant to be the highest. Hence, we can conclude that the presence of glycosides acts as a green stabilizer responsible for the durability of Mahī for so many centuries. One research article on 'Role of Biosurfactant in the binding of herbal ink Mahī: A view towards green chemistry’ is planned.

Again, another attempt was made to improve the traditional procedure at artificial setting under refrigerator instead of natural setting in cold condition. Different analytical tools were applied to study the effectiveness under artificial setting. Raman spectra showed intense peaks at $1590 \mathrm{~cm}^{-1}, 1352 \mathrm{~cm}^{-1}$ and $634 \mathrm{~cm}^{-1}$. Raman measurements on the optimized Mahi showed the presence of amorphous carbon (broad bands at 1334 and $1588 \mathrm{~cm}^{-1}$ ). The broadening of the band at 566 is attributed to the presence of other metallic salts. Powder-XRD analysis confirmed the presence $\mathrm{Fe}_{2} \mathrm{O}_{3} \cdot \mathrm{XH}_{2} \mathrm{O}$. Also, scanning electron microscopic image of Mahī stained on bond paper confirmed that there is no droplet of ink on the surface of bond paper. A full research paper on 'A physicochemical characterization of a medieval herbal ink, Mahī, of Assam, India' was published in the journal Coloration Technology, 134.6 (2018): 450-463, and two articles Sancipat, Mah̄̄ aru Hengul-Haitalr rasayanic dishto, and Mah̄̄ Asomor anupam bheshaj chhinyahi, in Sancipatiya puthi in Assamese have been published in a monograph HengulHaital in the year 2018. 


\subsection{Scientific study of Sancipat}

Some scientific study on preparation of Sancipat the writing base made of bark of Sanci tree was done by determining certain physicochemical parameters during its processing. Sanci tree bark (Aquilaria agallocha) grown locally were collected and writing-base using the traditional method were prepared. Various spectroscopic techniques like FT-IR, SEM-EDX, XRD, CHN were applied in the study. Some physicochemical parameters such as tensile strength, gloss index for making a comparison among Sancipat folios at different stages of preparation, viz., raw, degummed bark and finished Sancipat folio were determined. The antimicrobial study on the Sanci bark at different stages of Sancipat preparation was done.

From the study, it was observed that both tensile strength and gloss index get increased after undergoing the preparation steps as compared to raw Sanci. FT-IR study showed that all the spectra have uniform vibrational frequencies with an exception for the two namely base degummed Sanci and Sancipat, which shows a medium intensity for $-\mathrm{OH}$ stretching frequency (H-bonded). Again, for Sancipat, we observed a slight change in intensity for characteristic cellulose peak. In addition to this, from SEM images, we observed a smooth surface for Sancipat manuscript as compared to fibre like structure of raw Sanci. EDX study showed that the amount of carbon and oxygen slightly higher as compared to raw Sanci. XRD study proved the raw Sanci to be native cellulose $\left(\mathrm{C}_{6} \mathrm{H}_{12} \mathrm{O}_{6}\right) \mathrm{x}$. Finally, antimicrobial test showed that raw Sanci has no antifungal properties. However, Sanci treated with $\mathrm{CuSO}_{4}$, (hāitāl) and häitāl with hengul bears the potential of antifungual properties. This antifungal property of treated Sanci may be additive or synergistic or cumulative in nature. It is also possible that $\mathrm{CuSO}_{4}$, (hāitāl) and hāitāl with hengul might be responsible for the antifungal property of Sancipat manuscript.

The authors delivered a talk on 'Sancipat manuscript of Assam: revisiting traditional knowledge' at a national seminar on book history in NE India at Tezpur University during 12-13 February, 2019. A paper on 'Sanchipat, Mahī aru Hengul-Haital-ar antarkatha, was published in Sonit Prabha, mouthpiece of Tezpur Sahitya Sabha in 2019.

\subsection{Reviving the dyeing traditions of Sancipat and hengul-hāitāl}

A one-day workshop on 'the traditions of Sancipat manuscript and hengul-häitäl' was conducted on 11th September 2017 at Tezpur University. Around 30 traditional artisans, practicing the art participated and based on its recommendations, the Government of Assam is considering Sanci tree as an agricultural crop, in place of endangered species, which will promote its cultivation and the tradition of Sancipat manuscript.

Catalyzed by the present project, we took up and completed a work, during the winter of 2017-2018, involving restoration and renovation, in addition to scientific study, of some antique wood carvings of early nineteenth century at Batadrava Than, Nagaon, Assam, the birthplace of Shrimanta Sankardeva with traditional pigments (Figure 4). In addition to restoration of some precious medieval artifacts in traditional way, a group of 10 persons involved in related activities were also trained to take up such works independently. A similar restoration and renovation of some antique wood carvings in the form of workshops at Boralimora Sattra, Daparbatia, Tezpur was organized. The restoration work received a good media coverage and special praise from public (Figure 5, 6).

\section{Conclusion}

The scientific study of Mahi, revealed that some polyphenolic chemicals are responsible for the color of Mahi and some reasons for stability of the glaze of the ink for centuries. The study of the surface active property and the role of biosurfactants as green stabilizer on the chromogenic components of Mahi showed glycosides are responsible for its durability. During the scientific study on preparation of Sancipat, certain physicochemical parameters were determined during its processing.

\section{Bibliography}

[1] Gait E. A. A History of Assam, Thacker Spink \& Co, Kolkata, 1906.

[2] Kalita N. A Descriptive Catalogue of Manuscripts, Srimanta Sankardeva Research Institute, Batadrava, Nagaon, Assam, 1990. 

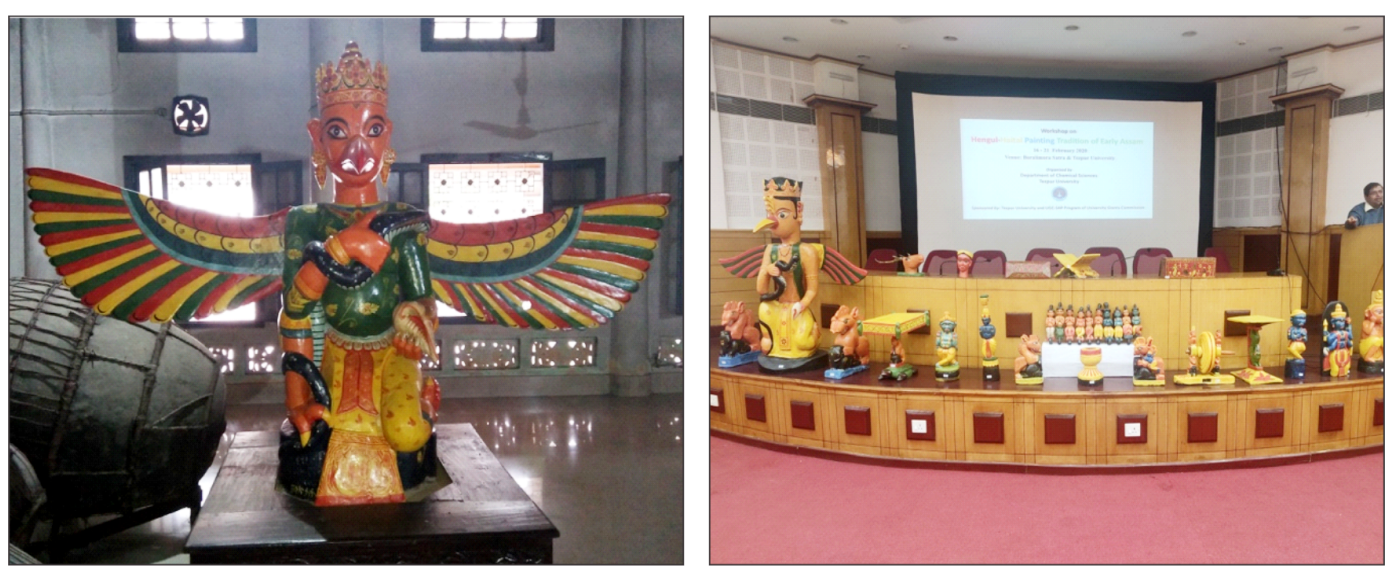

Figure 4 One of the four centuries-old woodcarvings restored at Batadrava Satra, Nagaon (left) and the centuries-old woodcarvings restored at Bor Ali Mora Satra, Tezpur (right).

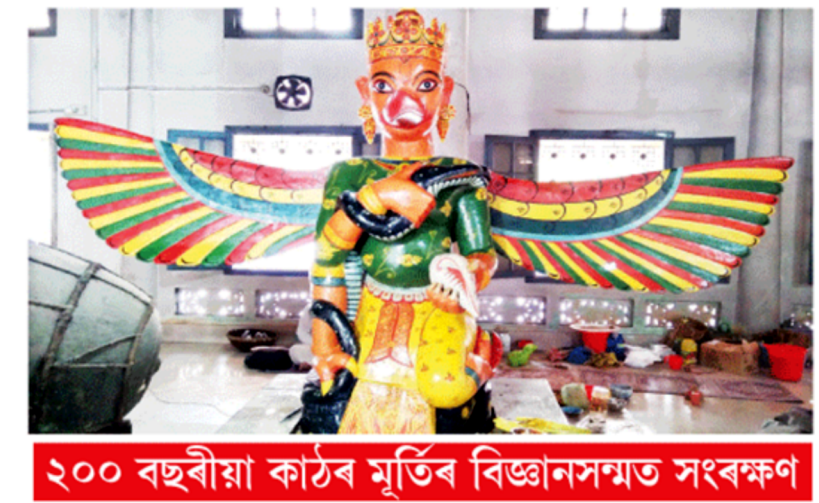

\section{জীরন্ত র্ পালে}

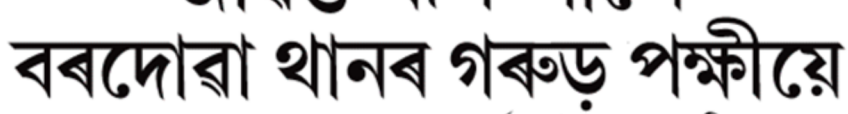

তেজপুব বিশ্ববিদ্যালয়ব প্রশংসনীয়

পদক্ষেপ, হেঙুল হাইতাল

বোলাই উজলালে প্রাচীন মূর্তি
বার্তা ব্যুব', 'তেজপুব, ৩ এপ্রিল ঃ মহাপুক্ব শ্রীমন্ত শংকবদেবব স্মৃতি বিজড়িত ববদোরা থানব কীর্তন घবব আপরুগীয়া কাঠব মৃর্তিসমৃহ সংবক্ষণব বিজ্ঞানসন্মত পদক্ষেপ লিছে তেজপুব বিশ্ববিদ্যালয় ১২ পৃষ্ঠাত
জীবন্ত ধপ পালে ববদোবা থানব

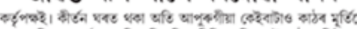

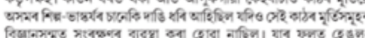

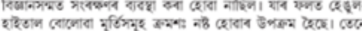

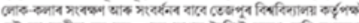

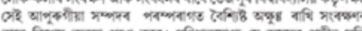

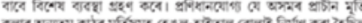

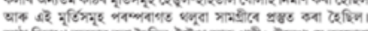

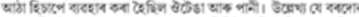

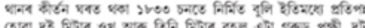
(2)

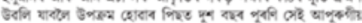

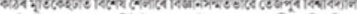

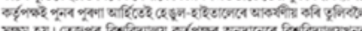

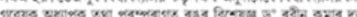

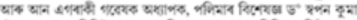

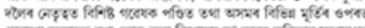

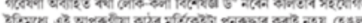

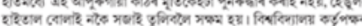

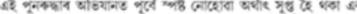

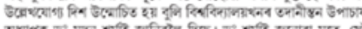

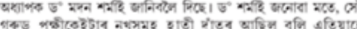
सरexp

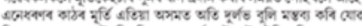

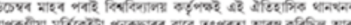

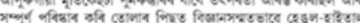

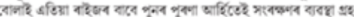

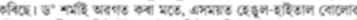

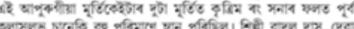

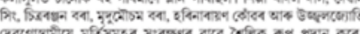

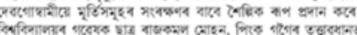

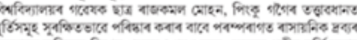

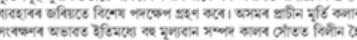

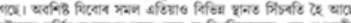

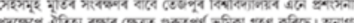

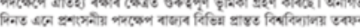

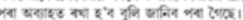

Figure 5 News clip in Dainik Asom, an Assamese daily on the restoration of woodcarving at Batadrava Than. 
The Telegraph, $5^{\text {th }}$ April, 2018

\section{Unique artwork in Xankardeb birthplace}

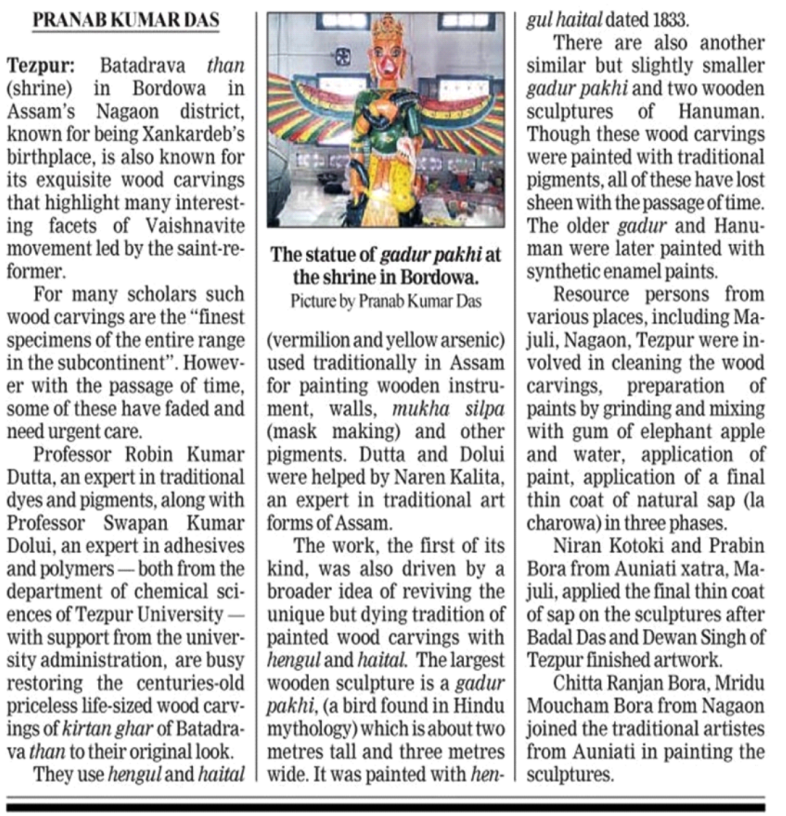

Figure 6 News clip published in The Telegraph on the restoration of woodcarving at Batadrava Than.

[3] Sah Anupam (ed). Indigenous Methods of Manuscript Preservation, National Mission for Manuscripts, New Delhi and D. K. Printworld (P), New Delhi, 2006.

[4] Nath D. (ed). Pre-modern writing culture in Assam: the tradition of manuscript writing. In, Religious Traditions and Social Practices in Assam, DVS Publishers, Guwahati, 2015.

[5] Dutta R. K. The science in the traditional manuscript-writing aids of Assam: Sancipat, Mahī and Hengul-Haital, In, Religious Traditions and Social Practices in Assam, DVS Publishers, Guwahati, 2015.

[6] Goswami B. R., Das M. K., Das P. P., Ramteke A., Medhi T. K., Hazarika S. and Dutta R. K. Mahī: A unique traditional ink of early Assam, Current Science, 112 (2017): 591-595.

[7] Goswami B. R., Chamuah N. and Dutta R. K. A physicochemical characterization of a medieval herbal ink, Mahī, of Assam, India, Color Technol., 134.6 (2018): 450-463.

[8] Dutta R. K., Sancipat, Mahī Aru Hengul-Haitalr Rasayanic Dishto, In Hengul Haital (Assamese) ed. Kalita N., Kolog Kala Kendra, Nagaon, 2018.

[9] Dutta R. K. and Goswami B. R., Mahī-Asomor Anupam Bheshaj Chhinyahi, In Sancipatiya Puthi (Assamese), ed. Kalita, N, Kolog Kala Kendra, Nagaon, 2018.

[10] Reviving Assam's ancient ink, The Hindu (all editions), Life Page, March, 20, 2017. 